\title{
Evaluation of the input site and characteristics of the antegrade fast pathway based on three-dimensional bi-atrial stimulus-ventricle mapping
}

\author{
Kazuhisa Matsumoto ${ }^{1}$ - Takeshi Tobiume ${ }^{1} \cdot$ Tomomi Matsuura $^{1} \cdot$ Takayuki Ise $^{1} \cdot$ Kenya Kusunose $^{1} \cdot$ \\ Koji Yamaguchi ${ }^{1}$. Shusuke Yagi ${ }^{1}$ - Daijyu Fukuda ${ }^{1}$ - Tetsuzo Wakatsuki ${ }^{1} \cdot$ Hirotsugu Yamada $^{1} \cdot$ Takeshi Soeki $^{1}$. \\ Masataka Sata ${ }^{1}$
}

Received: 31 March 2021 / Accepted: 22 June 2021 / Published online: 7 July 2021

(c) The Author(s) 2021

\begin{abstract}
Purpose Previous studies examined the right atrial (RA) input site of the antegrade fast pathway (AFp) (AFpI). However, the left atrial (LA) input to the atrioventricular (AV) node has not been extensively evaluated. In this study, we created three-dimensional (3-D) bi-atrial stimulus-ventricle (St-V) maps and analyzed the input site and characteristics of the AFp in both the RA and LA.

Methods Forty-four patients diagnosed with atrial fibrillation or WPW syndrome were included in this study. Three-dimensional bi-atrial St-V mapping was performed using an electroanatomical mapping system. Sites exhibiting the minimal St-V interval (MinSt-V) were defined as AFpIs and were classified into seven segments, four in the RA (F, S, M, and I) and three in the LA (M1, M2, and M3). By combining the MinSt-V in the RA and LA, the AFpIs were classified into three types: RA, LA, and bi-atrial (BA) types. The clinical and electrophysiological characteristics were compared.

Results AFpIs were most frequently observed at site S in the RA (34\%) and M2 in the LA (50\%), and the BA type was the most common (57\%). AFpIs in the LA were recognized in $75 \%$ of the patients. There were no clinical or electrophysiological indicators for predicting AFpI sites.

Conclusions Three-dimensional bi-atrial St-V maps could classify AFpIs in both the RA and LA. AFpIs in the LA were frequently recognized. There were no significant clinical or electrophysiological indicators for predicting AFpI sites, and 3-D bi-atrial St-V mapping was the only method to reveal the precise AFp input site.
\end{abstract}

Keywords Antegrade fast pathway $\cdot \mathrm{St}-\mathrm{V}$ map $\cdot \mathrm{St}-\mathrm{H}$ map $\cdot$ Left atrial input $\cdot$ Right atrial input

\begin{tabular}{|c|c|c|c|}
\hline \multicolumn{2}{|c|}{ Abbreviations } & IDF & Inferiorly dislocated AFp \\
\hline RA & Right atrium/right atrial & $\mathrm{Pi}$ & Pacing site \\
\hline LA & Left atrium/left atrial & TOK & Triangle of Koch \\
\hline BA & Bi-atrial & MA & Mitral annulus \\
\hline A-H & Atrial-His & TA & Tricuspid annulus \\
\hline $\mathrm{H}-\mathrm{V}$ & His-ventricle & St-H interval & Interval between the stimulation artifact \\
\hline AFp & Antegrade fast pathway & & and His bundle electrogram \\
\hline AFpI & Input site of AFp to the AVN & St-V interval & Interval between the stimulation artifact \\
\hline AFpI(RA) & AFpI in the RA & & and peak of the QRS wave in lead \\
\hline \multirow[t]{2}{*}{$\operatorname{AFpI}(\mathrm{LA})$} & AFpI in the LA & MinSt-V & The site showing the minimal St-V \\
\hline & & MinSt-V(RA) & MinSt-V in RA \\
\hline \multirow[t]{2}{*}{$凶$} & sumoto & MinSt-V(LA) & MinSt-V in LA \\
\hline & zuhisa@tokushima-u.ac.jp & VAT & Ventricular activation time (the time from \\
\hline $\begin{array}{rr}1 & \text { Departm } \\
& \text { Hospital }\end{array}$ & $\begin{array}{l}\text { Cardiology, Tokushima University } \\
-15 \text { Kuramoto-cho, Tokushima City, }\end{array}$ & & the $\mathrm{R}$ wave \\
\hline
\end{tabular}




\section{Introduction}

The exit site of the retrograde fast pathway (RFp) from the atrioventricular node (AVN) can be determined by mapping the earliest activation site in the atrium during constant pacing from the ventricle. On the other hand, the input site of the antegrade fast pathway (AFp) to the AVN has been very difficult to determine and only stimulus-His (St-H) mapping can identify the input site of the AFp to the AVN (AFpI) [1-5]. The use of a three-dimensional (3-D) mapping system makes identification of the exact location of the AFpI easier to determine [4, 5]. Previous studies, however, have examined the AFpI only in the right atrium (RA) (AFpI[RA]). The left atrial (LA) input to the AVN has not been extensively evaluated and only Gonzalez et al. have suggested that an AFpI exists in the LA (AFpI[LA]) with high probability in $95 \%$ of patients [6]. In this study, we created 3-D bi-atrial stimulus-ventricle (St-V) maps, which were equivalent to St-H maps, and analyzed the input site and characteristics of the AFp in both the RA and LA.

\section{Methods}

\subsection{Study population}

From June 1, 2019, to August 31, 2020, forty-four consecutive patients ( 31 men and 13 women, $64 \pm 14$ years, range 25 to 80 years) with atrial fibrillation (AF) (18 paroxysmal and 16 persistent) or Wolff-Parkinson-White (WPW) syndrome with a left free wall accessory pathway who underwent catheter ablation were included in this study. Patients with first degree atrioventricular block and left bundle branch block were excluded because an AFp might not exist in the former and because transient AV block during mapping might occur in the latter. The institutional review board of Tokushima University approved the study protocol. Written informed consent was obtained from all patients.

\subsection{Electrophysiological study}

Patients with atrial fibrillation were studied under deep sedation with a continuous infusion of propofol. They were on a ventilator and underwent continuous monitoring of the blood pressure, oxygen saturation, and bispectral index. Patients with WPW syndrome were studied under local anesthesia using xylocaine. Venous access was established percutaneously from the right jugular vein and right femoral vein to introduce electrode catheters into the RA, right ventricle (RV), coronary sinus (CS), and LA. A His bundle electrogram recording catheter (His) was used in the case of WPW syndrome, but not in the case of AF. All patients required a transseptal left heart catheterization for a pulmonary vein isolation or accessory pathway ablation. To record the electrocardiogram from the RA and CS, a 6-Fr catheter with 20 electrodes (BeeAT; Japan Lifeline, Tokyo) was inserted via the right jugular vein into the CS. A 5-Fr catheter with 5 electrodes (Arma, Century Medical Inc., Tokyo, Japan) was positioned in the RV apex (RVA). The CARTO®3 System (Biosense Webster, Irvine, California) was used for 3-D mapping in all patients. The bipolar electrocardiograms were filtered at 30-400 Hz for the electrophysiological analysis (CardioLab, GE Healthcare Japan, Tokyo).

\subsection{Identifying the input site of the antegrade fast pathway using 3-D bi-atrial St-V maps}

During the waiting period after the ablation, 3-D bi-atrial St-V maps for identifying the AFpI were created in all patients during constant atrial pacing at $100 \mathrm{ppm}(600 \mathrm{~ms})$ from a 7-Fr irrigation catheter (Thermocool SmartTouch SF, Biosense Webster). The pacing output was set at 3-4 V/1 ms in order to avoid capturing the AVN, His bundle, and ventricles. As shown in Fig. 1, constant pacing was performed at one pacing site (Pi) and, after reaching a steady state, the interval between the stimulation artifact and peak of the QRS wave in lead II (St-V interval) was measured. Data regarding both the location of the pacing site (Pi) and St-V interval at the Pi were stored on the 3-D map. Then, the pacing catheter was moved to the next site $(\mathrm{Pi}+1)$. The AFpI was defined as the site exhibiting the minimal St-V interval (MinSt-V). The AFpI[RA] and AFpI[LA] were defined as the MinSt-V in the RA (MinSt-V[RA]) and LA (MinSt-V[LA]), respectively. The sites where the His bundle electrocardiogram was recorded were also stored on the 3-D map during sinus rhythm to identify the apex of the triangle of Koch (TOK) without the electrocardiographic information, and the A-H interval during sinus rhythm was measured at those sites. The anatomical information related to the roof and floor of the CS ostium, tricuspid annulus, and mitral annulus were also stored on the 3-D map.

\subsection{Classification of the input site of the antegrade fast pathway}

As shown in Fig. 2, for the classification of the AFpI, the atrial septum was subdivided into seven segments, four on the RA aspect and three on the LA side. In the RA, the TOK was classified into three equidistant parts: superior, middle, and inferior thirds defined as sites S, M, and I, respectively. Also, the part posterior to the TOK was defined as site F. If the AFpI was located at site $\mathrm{M}$ or I, the AFpI was defined as an inferiorly dislocated AFp (IDF). In the LA, the AFpI was classified into three segments along the mitral annulus (MA): the 6-7 o' clock, 7-8 o'clock, and 8-9 o' clock directions along the mitral annulus were labeled as sites M1, M2, and M3, respectively. 


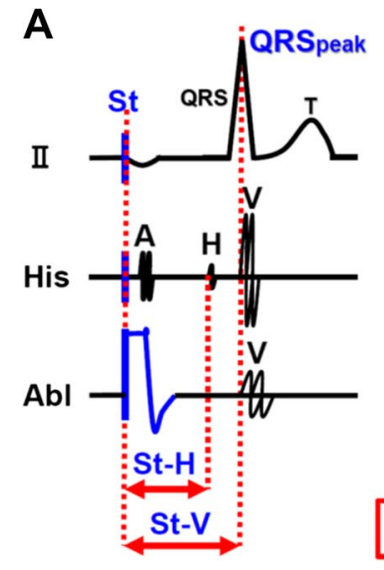

Fig. 1 Schematic presentation of the method of St-V mapping (intracardiac electrogram (A) and electroanatomical map (B)). (A) a: During constant pacing from Abl catheter, St-V interval, which means the conduction time between the stimulation artifact and the peak of QRS wave in lead II, was measured at each pacing site. Pacing output was set at 3-4 V/1 ms (B) St-V interval of the $i$ th pacing site was shown as "ai $+b+c$." At MinSt-V, ai was approximately zero and MinSt-V was almost identical with AFpI, where ai was equal to zero. His, His bundle catheter; Abl, ablation catheter; St, stimulus; A, atrial electro-

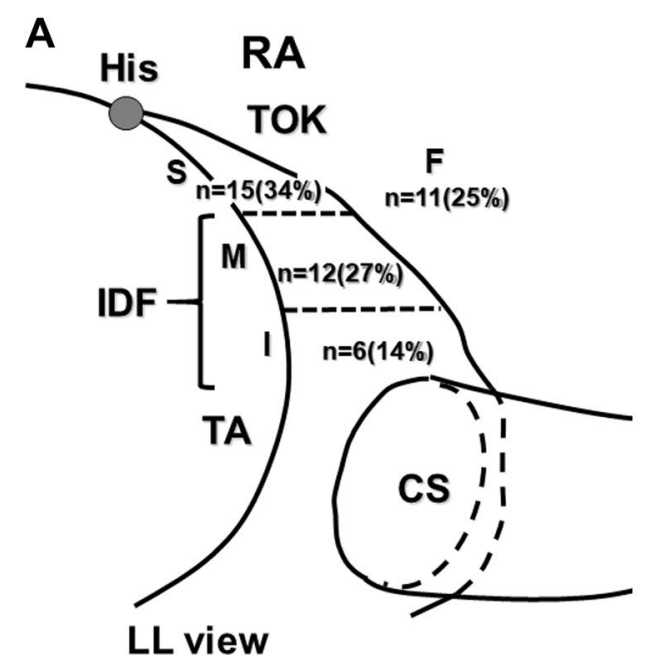

Fig. 2 Schematic presentation of the triangle of Koch (TOK) of the right atrium (RA) in the left lateral (LL) view (A) and the mitral annulus (MA) of left atrium (LA) in the left anterior oblique view (B). A: TOK was divided into 3 parts (superior third part, middle third part, and inferior third part were defined as site $\mathrm{S}$, site $\mathrm{M}$, and

\subsection{Classification of the type of input site of the antegrade fast pathway by combining the right and left atria}

As shown in Fig. 3, by combining the AFpI[RA] (MinSt$\mathrm{V}[\mathrm{RA}]$ ) and AFpI[LA] (MinSt-V[LA]) for the classification of the type of AFpI, the AFpI was classified into three gram; H, His bundle electrogram; V, ventricular electrogram; St-H, stimulus-His; St-V, stimulus-ventricle; AVN, atrioventricular node; $\mathrm{AFp}$, antegrade fast pathway; AFpI, input site of AFp to AVN; $\mathrm{Pi}$, $i$ th pacing site; $\mathrm{Pn}, n$th pacing site; $\mathrm{St}-\mathrm{H}$ interval(i), St-H interval of the $i$ th pacing site; St-V interval(i), St-V interval of the $i$ th pacing site; MinSt-V, the pacing site showing the minimal St-V interval; a, conduction time between the pacing site and AFpI; b, conduction time between $\mathrm{AFpI}$ and His bundle electrogram; c, conduction time between His bundle electrogram and the peak of QRS wave in lead II

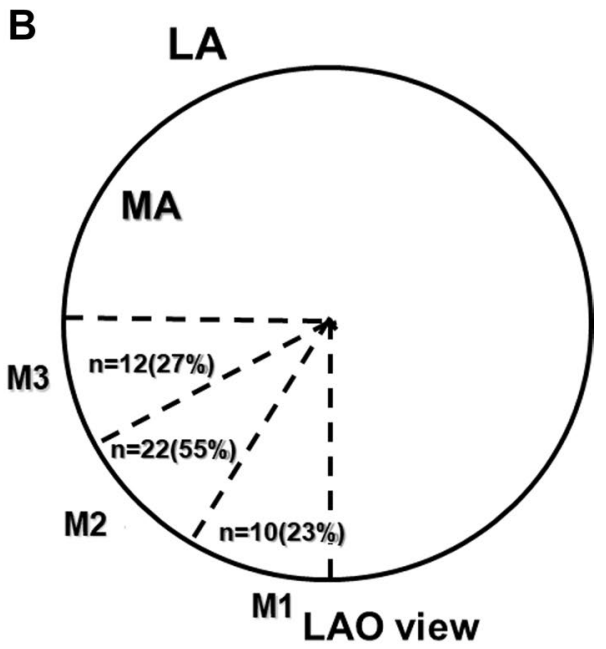

site I, respectively), and a part posterior to the TOK was defined as site F. B: MA was divided into 3 parts. The direction of 6-7, 7-8, and 8-9 o'clock of MA were defined as site M1, site M2, and site M3, respectively. His, His bundle electrogram recording site; TOK, triangle of Koch; IDF, inferiorly dislocated AFp

types: RA type, LA type, and bi-atrial (BA) type. The RA type was defined when the following two conditions were met: (1) the MinSt-V[RA] was shorter than that in the LA, and (2) there was no site on the shortest distance between the MinSt-V[RA $]$ and MinSt-V[LA] along the atrial septum where the St-V interval was longer than the MinSt-V[LA]. In the RA type, the MinSt-V[RA] was the only AFpI 

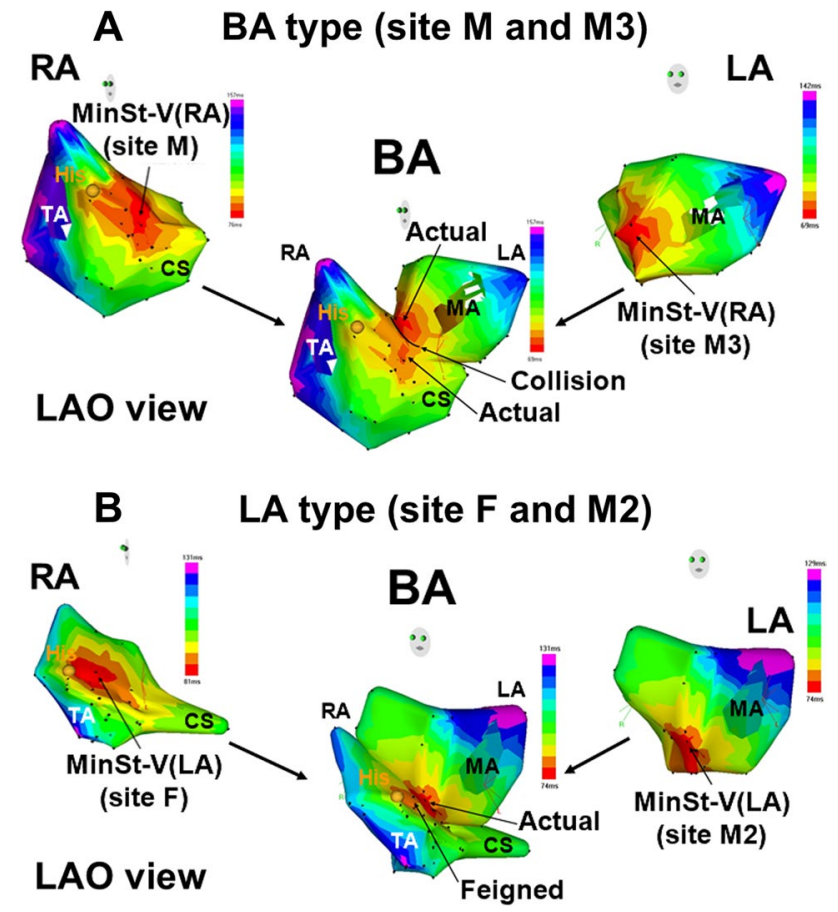

Fig. 3 Examples of AFpI of BA type (A) and LA type (B) using 3-D bi-atrial St-V map. A: In BA type of AFp, minimal St-V interval in RA and LA showed almost the same value. MinSt-V(RA) and MinStV(LA) were clearly separated with some sites on the shortest distance between MinSt-V(RA) and MinSt-V(LA) along the atrial septum where St-V interval was longer than both minimal St-V intervals in RA and LA. In BA type, both MinSt-V(RA) and MinSt-V(LA) represent two different AFpI (actual). B: In LA type of AFp, minimal St-V interval in LA showed smaller value than that in RA and there was no site on the shortest distance between MinSt-V(RA) and MinSt-V(LA) along the atrial septum where the St-V interval was longer than minimal St-V interval in RA. In LA type, MinSt-V(LA) represents the only AFpI (actual) while MinSt-V(RA) does not represent an AFpI (feigned)

(actual) and the MinSt-V[LA] was not the AFpI (feigned). An LA type was defined when the following two conditions were met: (1) the MinSt-V[LA] was shorter than that in the RA, and (2) there were no sites on the shortest distance between the MinSt-V[RA] and MinSt-V[LA] along the atrial septum where the St-V interval was longer than the MinSt-V[RA]. In the LA type, the MinSt-V[LA] was the only AFpI (actual) and the MinSt-V[RA] was not the AFpI (feigned). A BA type was defined when the following two conditions were met: (1) the MinSt-V[RA] and MinStV[LA] exhibited almost the same value, and (2) there were some sites on the shortest distance between the MinSt$\mathrm{V}[\mathrm{RA}]$ and MinSt-V[LA] along the atrial septum where the St-V interval was longer than both the MinSt-V[RA] and MinSt-V[LA]. In the BA type, the MinSt-V[RA] and MinSt-V[LA] were two separate AFpIs (actual).

\subsection{Statistical analysis}

Data are expressed as the mean \pm SD. Differences between the means were compared using a one-way analysis of variance or the Kruskal-Wallis test. A Levene's test was used to check for the equality of variance. The differences in the proportions were compared using the chi-square test. A $P<0.05$ was considered statistically significant. All statistical analyses were performed with the Statistical Package for the Social Sciences for Windows software (SPSS, version 27, Chicago, IL, USA).

\section{Results}

\subsection{Clinical and electrophysiological characteristics of the study population}

As shown in Table 1, 66\% (29/44) of the patients in this study population continued antiarrhythmic drugs during the procedure. In patients with WPW syndrome, the antiarrhythmic drugs were discontinued preoperatively. Twenty-nine of 34 patients with AF continued on antiarrhythmic drugs. Class I drugs were used in 13 patients (cibenzoline 7, pilsicainide 5 , and propafenone 1), class II (bisoprolol) in 7 patients, and class IV in 9 patients (verapamil 4 and bepridil 5). No class III drugs were used. The mean left atrial volume index (LAVI) was $40 \pm 14 \mathrm{~mL} / \mathrm{m}^{2}$. The A-H interval during sinus rhythm was $94 \pm 16 \mathrm{~ms}$. The number of mapping points was $29 \pm 9$ points in the RA and $25 \pm 10$ points in the LA. The minimal St-V interval was $162 \pm 45 \mathrm{~ms}$ in the RA and $161 \pm 42 \mathrm{~ms}$ in the LA.

\subsection{Location of the input site of the antegrade fast pathway in the RA}

As shown in Fig. 2 and Table 2, the MinSt-V[RA] was observed at site $\mathrm{F}$ in 11 patients (25\%), site $\mathrm{S}$ in $15(34 \%)$, site $\mathrm{M}$ in $12(27 \%)$, and site I in $6(14 \%)$. An IDF was observed in $41 \%$ of the patients. There were no significant differences among these four groups with regard to the age, gender, type of arrhythmia, LAVI, A-H interval during sinus rhythm, minimal St-V interval, and site of the MinSt-V[LA]. The minimal distance between the His bundle electrogram recording site and MinSt-V[RA] did not correlate with the A-H interval during sinus rhythm (Fig. 4).

\subsection{Location of the input site of the antegrade fast pathway in the LA}

As shown in Fig. 2 and Table 3, the MinSt-V[LA] was observed at site M1 in $10(23 \%)$, site M2 in $22(50 \%)$, 
Table 1 Clinical and electrophysiological characteristics of the study population

\begin{tabular}{ll}
\hline & $n=44$ \\
\hline Age (years) (mean \pm SD) & $64 \pm 14$ \\
Male/female ratio & $31 / 13$ \\
Type of arrhythmia & \\
Paroxymal AF/persistent AF/WPW syndrome & $18 / 16 / 10$ \\
Continuation of antiarrhythmic drugs $(n(\%))$ & $29(66)$ \\
Vaughan Williams classification (I/II/III/IV) & $13 / 7 / 0 / 9$ \\
LAVI (ml/m²) (mean \pm SD) & $40 \pm 14$ \\
A-H interval during sinus rhythm (ms) (mean \pm SD) & $94 \pm 16$ \\
Number of mapping points (mean \pm SD) & \\
RA/LA & $29 \pm 9 / 25 \pm 10$ \\
Minimal St-V interval (mean \pm SD) & $162 \pm 45 / 161 \pm 42$ \\
RA/LA & $11 / 8 / 25$ \\
Classification of the AFp (RA type/LA type/BA type) & $15 / 12 / 6 / 11$ \\
Site of the minimal St-V interval in the RA (S/M/I/F) & $10 / 22 / 12$ \\
Site of the minimal St-V interval in the LA (M1/M2/M3) & \\
\hline
\end{tabular}

$S D$, standard deviation

Table 2 Clinical and electrophysiological characteristics of the sites showing the minimal St-V interval in the RA

\begin{tabular}{|c|c|c|c|c|c|c|}
\hline & & Site $\mathrm{F}(n=11)$ & Site $S(n=15)$ & Site $M(n=12)$ & Site $\mathrm{I}(n=6)$ & $p$ value \\
\hline Age (years) $($ mean $\pm \mathrm{SD})$ & & $69 \pm 7$ & $67 \pm 9$ & $64 \pm 14$ & $49 \pm 28$ & 0.112 \\
\hline Male/female ratio & & $8 / 3$ & $9 / 6$ & $9 / 3$ & $5 / 1$ & 0.703 \\
\hline \multicolumn{7}{|l|}{ Type of arrhythmia } \\
\hline Paroxysmal AF/persistent AF/WPW syndrome & & $3 / 6 / 2$ & $7 / 6 / 2$ & $6 / 4 / 2$ & $2 / 0 / 4$ & 0.117 \\
\hline $\mathrm{LAVI}\left(\mathrm{ml} / \mathrm{m}^{2}\right)($ mean $\pm \mathrm{SD})$ & & $48 \pm 17$ & $39 \pm 11$ & $37 \pm 15$ & $28 \pm 6$ & 0.155 \\
\hline A-H interval during sinus rhythm $(\mathrm{ms})($ mean $\pm \mathrm{SD})$ & & $89 \pm 14$ & $96 \pm 22$ & $95 \pm 14$ & $97 \pm 13$ & 0.651 \\
\hline \multirow[t]{2}{*}{ Minimal St-V interval (ms) (mean \pm SD) } & RA & $161 \pm 50$ & $174 \pm 30$ & $162 \pm 46$ & $149 \pm 65$ & 0.387 \\
\hline & LA & $155 \pm 48$ & $170 \pm 27$ & $170 \pm 42$ & $145 \pm 60$ & 0.313 \\
\hline Site of the minimal St-V interval in the LA (M1/M2/M3) & & $1 / 7 / 3$ & $4 / 7 / 4$ & $5 / 3 / 4$ & $0 / 5 / 1$ & 0.242 \\
\hline
\end{tabular}

and site M3 in 12 (27\%). There were no significant differences among these three groups with regard to the age, gender, type of arrhythmia, LAVI, A-H interval during sinus rhythm, minimal St-V interval, and site of the MinSt$\mathrm{V}$ [RA]. Interestingly, only sites $\mathrm{F}$ and $\mathrm{S}$ were recognized in the LA type. The minimal distance between the His bundle electrogram recording sites and MinSt-V(LA) was not correlated with the A-H interval during sinus rhythm (Fig. 4).

\subsection{Type of the input site of the antegrade fast pathway by combining the right and left atria}

As shown in Table 3, the AFpI was classified as an RA type in $11(25 \%)$, LA type in 8 (18\%), and BA type in 25 (57\%). There were no significant differences among these three groups with regard to the age, gender, type of arrhythmia, LAVI, A-H interval during sinus rhythm, minimal St-V interval, site of the MinSt-V[RA], and site of the minimal St-V interval in the LA. Examples of the AFpI in the BA type and LA type are shown in Fig. 3. Figure 3A shows an example of a BA type, and the MinSt-V[RA] was site $\mathrm{M}$ and MinSt-V[LA] site M3. The MinSt-V[RA] and MinStV[LA] were $76 \mathrm{~ms}$ and $69 \mathrm{~ms}$, respectively. There were some sites on the shortest distance between the MinSt-V[RA] and MinSt-V[LA] along the septal wall where the St-V interval was longer than both the MinSt-V[RA] and MinSt-V[LA], and the 3-D bi-atrial St-V map exhibited two clearly separate AFpIs. Figure 3B shows an example of an LA type, and the MinSt-V[RA] was site F and MinSt-V[LA] site M2. The MinSt-V[RA] and MinSt-V[LA] were $81 \mathrm{~ms}$ and $74 \mathrm{~ms}$, respectively. There was no site on the shortest distance between the MinSt-V[RA] and MinSt-V[LA] along the atrial wall where the St-V interval was longer than the MinSt-V[RA], and the 3-D bi-atrial St-V map exhibited only one AFpI. 
Fig. 4 Relationship between A-H interval during sinus rhythm and the minimal distance between His and AFpI(MinSt-V) in RA. There was no relationship between A-H interval during sinus rhythm and the minimal distance between His and AFpI (MinSt-V) in RA and LA. $p=0.906(\mathrm{RA}), p=0.606(\mathrm{LA})$

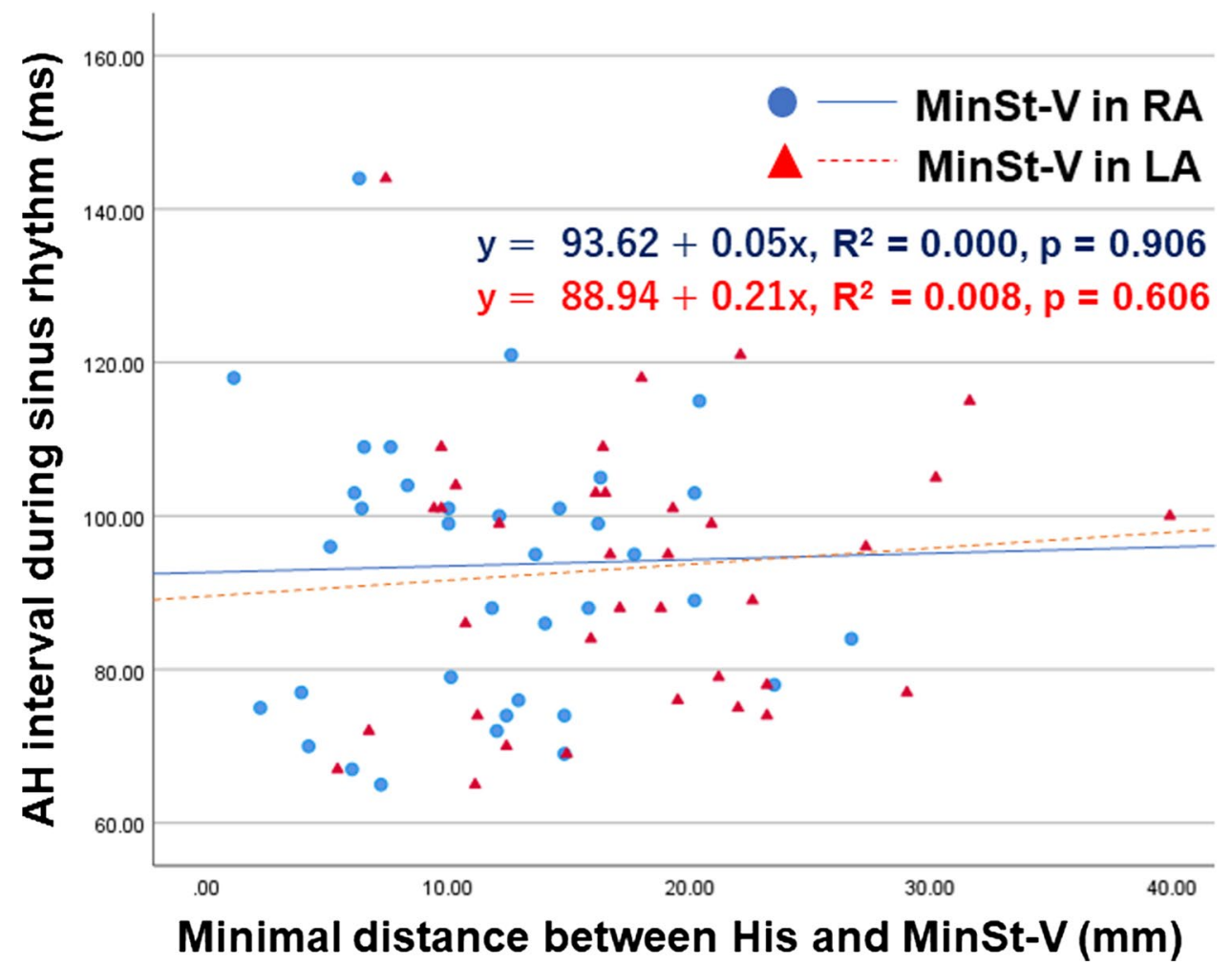

Table 3 Clinical and electrophysiological characteristics of the sites with the minimal St-V interval in the LA

\begin{tabular}{|c|c|c|c|c|c|}
\hline & & Site M1 $(n=10)$ & Site M2 $(n=22)$ & Site M3 ( $n=12)$ & $p$ value \\
\hline Age (years) (mean \pm SD) & & $66 \pm 10$ & $68 \pm 12$ & $56 \pm 17$ & 0.06 \\
\hline Male/female ratio & & $9 / 1$ & $14 / 8$ & $8 / 4$ & 0.3 \\
\hline \multicolumn{6}{|l|}{ Type of arrhythmia } \\
\hline Paroxysmal AF/persistent AF/WPW syndrome & & $5 / 3 / 2$ & $7 / 9 / 6$ & $6 / 4 / 2$ & 0.812 \\
\hline $\mathrm{LAVI}\left(\mathrm{ml} / \mathrm{m}^{2}\right)($ mean $\pm \mathrm{SD})$ & & $31 \pm 11$ & $45 \pm 16$ & $38 \pm 12$ & 0.08 \\
\hline A-H interval during sinus rhythm $(\mathrm{ms})($ mean $\pm \mathrm{SD})$ & & $95 \pm 14$ & $98 \pm 17$ & $86 \pm 15$ & 0.094 \\
\hline \multirow[t]{2}{*}{ Minimal St-V interval $(\mathrm{ms})($ mean $\pm \mathrm{SD})$} & RA & $170 \pm 42$ & $161 \pm 51$ & $157 \pm 40$ & 0.547 \\
\hline & LA & $166 \pm 40$ & $160 \pm 50$ & $161 \pm 31$ & 0.784 \\
\hline Site of the minimal St-V interval in the RA (F/S/M/I) & & $1 / 4 / 5 / 0$ & $7 / 7 / 3 / 5$ & $3 / 4 / 4 / 1$ & 0.242 \\
\hline
\end{tabular}

\section{Discussion}

\subsection{Major findings}

In the present study, using the 3-D bi-atrial St-V map, it was possible to classify the input sites of the AFp in both the RA and LA. An AFpI[LA] was frequently observed and was recognized in $75 \%$ of the patients. The AFpI[LA] and AFpI[RA] were most frequently observed at site M2 (50\%) and site $\mathrm{S}(34 \%)$, respectively. The A-H interval during sinus rhythm was not correlated with the minimal distance between the His bundle electrogram recording sites and the MinSt-V[RA] and MinSt-V[LA] (Table 4).

\subsection{St-V map as an alternative to an St-H map}

In the previous studies, the $\mathrm{St}-\mathrm{H}$ map was the standard map used for the determination of the AFpI [1-5]. In this study, an St-V map was used instead of the St-H map because a His catheter was not used in the majority of the patients who had atrial fibrillation $(77 \%)$ and the St-V map was equivalent to the St-H map. As shown in Fig. 1, the St-V interval $(a \mathrm{a}+\mathrm{b}+\mathrm{c})$ was equal to the interval that consisted of the St-H interval $(\mathrm{ai}+\mathrm{b})$ and interval between the onset of the His bundle electrogram and peak of the QRS wave in lead II (c). Here, the interval between the onset of the His bundle electrogram and peak of the QRS wave in lead II (c) was equivalent to the sum of the H-V interval and 
Table 4 Clinical and electrophysiological characteristics of the RA, LA, and BA type

\begin{tabular}{|c|c|c|c|c|c|}
\hline & & RA type $(n=11)$ & LA type $(n=8)$ & BA type $(n=25)$ & $p$ value \\
\hline Age (years) $($ mean $\pm S D)$ & & $65 \pm 14$ & $70 \pm 7$ & $62 \pm 15$ & 0.325 \\
\hline Male/female ratio & & $10 / 1$ & $4 / 4$ & $17 / 8$ & 0.143 \\
\hline \multicolumn{6}{|l|}{ Type pf arrhythmia } \\
\hline Paroxysmal AF/persistent AF/WPW syndrome & & $4 / 3 / 4$ & $4 / 3 / 1$ & $10 / 10 / 5$ & 0.751 \\
\hline $\mathrm{LAVI}\left(\mathrm{ml} / \mathrm{m}^{2}\right)($ mean $\pm \mathrm{SD})$ & & $39 \pm 13$ & $37 \pm 9$ & $41 \pm 16$ & 0.812 \\
\hline A-H interval during sinus rhythm $(\mathrm{ms})($ mean $\pm \mathrm{SD})$ & & $97 \pm 20$ & $104 \pm 12$ & $90 \pm 15$ & 0.094 \\
\hline \multirow[t]{2}{*}{ Minimal St-V interval $(\mathrm{ms})($ mean $\pm \mathrm{SD})$} & RA & $142 \pm 48$ & $183 \pm 50$ & $165 \pm 41$ & 0.148 \\
\hline & LA & $157 \pm 45$ & $159 \pm 46$ & $163 \pm 42$ & 0.899 \\
\hline Site of the minimal St-V interval in the RA (F/S/M/I) & & $1 / 4 / 3 / 0$ & $3 / 4 / 0 / 0$ & $6 / 7 / 7 / 5$ & 0.146 \\
\hline Site of the minimal St-V interval in the LA (M1/M2/M3) & & $3 / 5 / 3$ & $2 / 6 / 0$ & $5 / 11 / 9$ & 0.366 \\
\hline
\end{tabular}

ventricular activation time (VAT: the time from the onset of the QRS wave to the peak of the R wave). The H-V interval and VAT are reported to have a very high reproducibility with minimal variability [7-10]. Therefore, the St-V map was considered an alternative to the St-H map.

\subsection{Left antegrade fast pathway input to the atrioventricular node}

To the best of our knowledge, this study was the first to investigate the detailed input site of the AFp in the LA. The 3-D bi-atrial St-V mapping with the CARTO system in this study revealed that an AFpI[LA] was present in $75 \%$ of the patients and was the only AFpI in $18 \%$. In the previous reports, an AFpI[LA] was suggested in cases with a left posteroseptal accessory pathway ablation $[11,12]$ or left-sided fast pathway ablation for typical atrioventricular nodal reentrant tachycardia (AVNRT) [13]. Moreover, the low success rate (46-69\%) of a right-sided fast pathway ablation for AVNRT reported by Mitrani et al. may also suggest the residual presence of an intact AFpI[LA] after an AFpI[RA] ablation [14]. The only report about the probable presence of an AFpI[LA], by Gonzalez et al., suggested that an AFpI[LA] existed with high probability in $95 \%$ of the patients [6]. That probability of the presence of an AFpI[LA] was higher than that in this study (75\%), which was because atrial extrastimulation to identify the $\mathrm{AFpI}[\mathrm{LA}]$ was used in their report.

\subsection{Right antegrade fast pathway input to the atrioventricular node}

The 3-D bi-atrial St-V mapping with the CARTO system in this study revealed the presence of an AFpI[RA] in $82 \%$ of the patients and only an AFpI in $25 \%$. An inferiorly dislocated AFp (IDF) was recognized in $41 \%$ (18/44) of the patients. The incidence of an IDF in this study was much higher than that reported by Delise et al. (10\%) [2], but was similar to that reported in the other Japanese studies $(33-56 \%)$ [3, 4]. These differences might be related to the ethnic differences and therefore, further studies will be needed.

\subsection{Clinical and electrophysiological indicators of the input site of the antegrade fast pathway}

In this study, the clinical and electrophysiological indicators including the age, gender, type of arrhythmia, LAVI, A-H interval during sinus rhythm, minimal St-V interval, and site of the MinSt-V[RA] had no significant discriminating ability regarding the input sites of the AFp. Therefore, pending further studies, St-V or St-H mapping remains the only method for identifying the precise location of the AFpI.

\subsection{Study limitations}

(1) This study was conducted at a single center and included only a small number of patients.

(2) Antiarrhythmic drugs were continued in many patients (66\%) because the majority of the patients studied had AF. Therefore, the St-V interval and other electrophysiological parameters could have been affected by those drugs.

(3) Two different diseases, AF and WPW, were included in this study, and all AF patients were studied under deep sedation and all WPW patients under local anesthesia. Therefore, the difference in the disease and effect of the sedation could have affected the electrophysiological parameters. Further studies are needed to evaluate the effects of those.

(4) In this study, we did not compare the effect of isoprenaline loading. The possibility that the input site of the 
AFp could change according to the isoprenaline loading remained.

(5) We fixed the pacing cycle length at $600 \mathrm{~ms}$ in order to evaluate the AFp, to overdrive the sinus rate, and to avoid Wenckebach periodicity. However, keeping a uniform pacing rate might have led us to overlook other concealed AV pathways.

(6) In this study, the St-V map was not compared with the St-H map. Although further studies will be needed to confirm this, it is reasonable to assume that the two maps would be equivalent.

\section{Conclusion}

The 3-D bi-atrial St-V map could classify the input site of the AFp in both the RA and LA. An AFpI[LA] was frequently present and was recognized in $75 \%$ of the patients. Except for the 3D bi-atrial St-V mapping, there were no significant clinical or electrophysiological indicators that could reveal the precise input site of the AFp.

Acknowledgements The authors thank Mr. John Martin for his linguistic assistance with this manuscript.

\section{Compliance with ethical standards}

Conflict of interest The authors have no conflict of interest. This study was approved by the institutional review board (IRB) of Tokushima University, and the IRB approval number was 3851.

Open Access This article is licensed under a Creative Commons Attribution 4.0 International License, which permits use, sharing, adaptation, distribution and reproduction in any medium or format, as long as you give appropriate credit to the original author(s) and the source, provide a link to the Creative Commons licence, and indicate if changes were made. The images or other third party material in this article are included in the article's Creative Commons licence, unless indicated otherwise in a credit line to the material. If material is not included in the article's Creative Commons licence and your intended use is not permitted by statutory regulation or exceeds the permitted use, you will need to obtain permission directly from the copyright holder. To view a copy of this licence, visit http://creativecommons.org/licenses/by/4.0/.

\section{References}

1. Delise P, Bonso A, Coro L, Fantinel M, Gasparini G, Themistoclakis S, Mantovan R. Pacemapping of the triangle of Koch: a simple method to reduce the risk of atrioventricular block during radiofrequency ablation of atrioventricular node reentrant tachycardia. Pacing Clin Electrophysiol. 2001;24:1725-31.
2. Delise P, Sitta N, Bonso A, Coro' L, Fantinel M, Mantovan R, Sciarra L, Zoppo F, Verlato R, Marras E, D'Este D. Pace mapping of Koch's triangle reduces risk of atrioventricular block during ablation of atrioventricular nodal reentrant tachycardia. J Cardiovasc Electrophysiol. 2005;16:30-5.

3. Tanaka Y, Yamabe H, Morihisa K, Uemura T, Kawano H, Nagayoshi Y, Kojima S, Ogawa H. Incidence and mechanism of dislocated fast pathway in various forms of atrioventricular nodal reentrant tachycardia. Circ J. 2007;71:1099-106.

4. Tanaka S, Yoshida A, Fukuzawa K, Takei A, Kanda G, Takami K, Kumagai H, Takami M, Itoh M, Imamura K, Fujiwara R, Hirata $K$. Recognition of inferiorly dislocated fast pathways guided by three-dimensional electro-anatomical mapping. J Interv Card Electrophysiol. 2011;32:95-103.

5. Suzuki A, Yoshida A, Takei A, Fukuzawa K, Kiuchi K, Tanaka S, Itoh M, Imamura K, Fujiwara R, Nakanishi T, Yamashita S, Matsumoto A, Konishi H, Ichibori H, Hirata K. Visualization of the antegrade fast and slow pathway inputs in patients with slow-fast atrioventricular nodal reentrant tachycardia. Pacing Clin Electrophysiol. 2014;37(7):874-83.

6. Gonzalez MD, Contreras LJ, Cardona F, Klugewicz CJ, Conti JB, Curtis AB, Morey TE, Dennis DM. Demonstration of a left atrial input to the atrioventricular node in humans. Circulation. 2002;106:2930-4.

7. Reddy CP, Damato AN, Akhtar M, Ogunkelu JB, Caracta AR, Ruskin JN, Lau SH. Time dependent changes in the functional properties of the atrioventricular conduction system in man. Circulation. 1975;52(6):1012-22.

8. Akhtar M, Damato AN, Batsford WP, Ruskin JN, Ogunkelu JB. A comparative analysis of antegrade and retrograde conduction patterns in man. Circulation. 1975;52(5):766-78.

9. Denker S, Lehmann M, Mahmud R, Gilbert C, Akhtar M. Effects of alternating cycle lengths on refractoriness of the His-Purkinje system. J Clin Invest. 1984;74(2):559-70.

10. Chilson DA, Zipes DP, Heger JJ, Browne KF, Prystowsky EN. Functional bundle branch block: discordant response of right and left bundle branches to changes in heart rate. Am J Cardiol. 1984;54(3):313-6.

11. Liu J, Dole LR. Late complete atrioventricular block complicating radiofrequency catheter ablation of a left posteroseptal accessory pathway. Pacing Clin Electrophysiol. 1998;21(11 Pt 1):2136-8.

12. Seidl K, Hauer B, Zahn R, Senges J. Unexpected complete AV block following transcatheter ablation of a left posteroseptal accessory pathway. Pacing Clin Electrophysiol. 1998;21(11 Pt 1):2139-42.

13. Kobza R, Hindricks G, Tanner H, Kottkamp H. Left-septal ablation of the fast pathway in AV nodal reentrant tachycardia refractory to right septal ablation. Europace. 2005;7(2):149-53.

14. Mitrani RD, Klein LS, Hackett FK, Zipes DP, Miles WM. Radiofrequency ablation for atrioventricular node reentrant tachycardia: comparison between fast (anterior) and slow (posterior) pathway ablation. J Am Coll Cardiol. 1993;21(2):432-41.

Publisher's note Springer Nature remains neutral with regard to jurisdictional claims in published maps and institutional affiliations. 